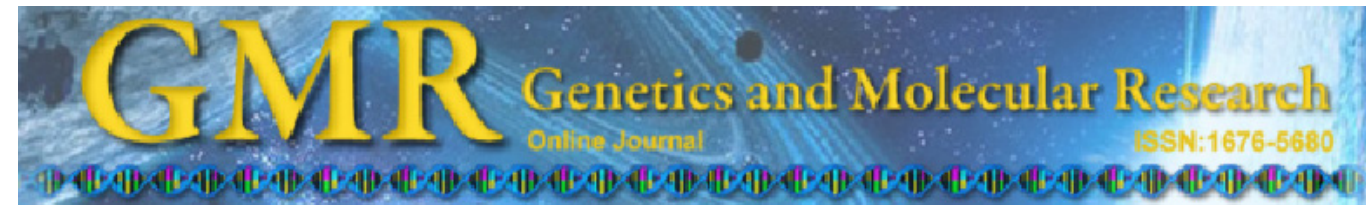

$\underline{\text { Short Communication }}$

\title{
Isolation and characterization of microsatellite markers for the Korean rockfish, Sebastes schlegeli
}

\author{
C.-C. Bai ${ }^{1,2}$, S.-F. Liu' ${ }^{2}$ Z.-M. Zhuang' ${ }^{2}$ L. Lin ${ }^{2,3}$, H. Wang ${ }^{1,2}$ and \\ Y.-Q. Chang ${ }^{1}$
}

${ }^{1}$ Key Laboratory of Mariculture, Ministry of Agriculture, Dalian Ocean University, Dalian, China

${ }^{2}$ Key Laboratory for Fishery Resources and Eco-Environment, Yellow Sea Fisheries Research Institute, Chinese Academy of Fishery Sciences, Qingdao, Shandong Province, China

${ }^{3}$ College of Oceanography and Environmental Science, Xiamen University, Xiamen, China

Corresponding author: S.-F. Liu

E-mail: liusf@ysfri.ac.cn

Genet. Mol. Res. 10 (3): 2065-2068 (2011)

Received June 15, 2011

Accepted August 10, 2011

Published September 15, 2011

DOI http://dx.doi.org/10.4238/vol10-3gmr1522

\begin{abstract}
The Korean rockfish (Sebastes schlegeli) is an important commercial fish that is widely used in aquaculture. We isolated and characterized 18 polymorphic microsatellite loci from the Korean rockfish using a (GT) $)_{13}$-enriched genomic library. Polymorphism was assessed in 48 individuals from a single population collected from the northern coastal waters of the Yellow Sea. The observed and expected heterozygosities ranged from 0.0244 to 0.7660 (mean 0.4194 ) and 0.0244 to 0.8758 (mean 0.5002 ), respectively. Polymorphism at these loci indicated from two to 15 alleles (mean 5.7); 14 of 18 loci conformed to Hardy-Weinberg equilibrium. These markers should be useful for management and conservation studies of this species.
\end{abstract}

Key words: Korean rockfish; Sebastes schlegeli; Genetic structure; Microsatellite loci 


\section{INTRODUCTION}

The Korean rockfish (Sebastes schlegeli) is the eurythermal, benthonic and semi-sedentary fish in the west of the North Pacific Ocean. It is also an important commercial fish in China, Korean and Japan (Hu et al., 2010). Overexploitation and environmental changes have caused a decline in Korean rockfish resources. Stock enhancement programs should be implemented to enrich fishery resources. It was reported that $S$. schlegeli has been listed as a mariculture and stock enhancement species in Korea at the beginning of 1990s (An and Park, 2009; Li, 2009).

It is important to estimate population genetic structure and genetic diversity to provide information for the conservation and management of S. schlegeli (Schwartz et al., 2007; Kitada et al., 2009; An and Park, 2009). Microsatellite markers are effective molecular markers that have been widely applied to management and conservation of this species because of the high level of polymorphism, co-dominant inheritance, and high abundance (Litt and Luty, 1989; Zhan et al., 2005, 2009). Six and fourteen microsatellite markers from $S$. schlegeli have already been isolated by Yoshida et al. (2005) and An and Park (2009), respectively. However, more microsatellite markers are needed for studying pedigree and building a genetic map. Here, we developed 18 polymorphic microsatellite markers for $S$. schlegeli.

\section{MATERIAL AND METHODS}

Forty-eight individuals of $S$. schlegeli were collected from the northern coastal waters of the Yellow Sea and preserved in alcohol until DNA extraction. Genomic DNA was extracted from muscle tissue using the phenol-chloroform procedure (Sambrook and Russell, 2001). Genomic DNA was simultaneously digested with MseI (New England Biolabs, USA), and the digested DNA $(10 \mu \mathrm{L})$ was ligated to $M s e I$ adaptors (5'-TACTCAGGAACTCAT-3'/5'GACGATGAGTCCTGAG-3'). Linker-ligated DNA was amplified in a $50-\mu \mathrm{L}$ reaction mix using the adaptor-specific primer (5'-GATGAGTCCTGAGTAA-3'). Polymerase chain reaction (PCR) conditions were as follows: 20 cycles at $94^{\circ} \mathrm{C}$ for $30 \mathrm{~s}, 53^{\circ} \mathrm{C}$ for $1 \mathrm{~min}, 72^{\circ} \mathrm{C}$ for $1 \mathrm{~min}$. The PCR products were purified using DNAmate (TaKaRa, Japan) and hybridized to a biotinlabeled $(\mathrm{GT})_{13}$ probe. The mixture was denatured at $94^{\circ} \mathrm{C}$ for $5 \mathrm{~min}$, then at $53^{\circ} \mathrm{C}$ for $15 \mathrm{~min}$. The hybrids were captured with streptavidin-coated magnetic beads (Promega, USA). Unhybridized DNA was washed away, and the remaining DNA was eluted from the magnetic beads and amplified using the adaptor-specific primer and the above program. Following purification, DNA fragments ranging from 500 to $1000 \mathrm{bp}$ were selected by separation on $1.5 \%$ agarose gels. The fragments were ligated to pMD18-T vectors (TaKaRa), and transformed into Escherichia coli DH5 $\alpha$ competent cells to construct an enriched microsatellite sequence library. After amplifying with (GT) $)_{10}$ and M13 primers, 192 positive clones were obtained. The positive clones were sequenced on an ABI 3730 automated DNA sequencer (Applied Biosystems, USA).

Primers were designed for 80 microsatellite-containing clones using PRIMER PREMIER5 (Premier Biosoft International, USA) and tested for polymorphism from six Sebastes schlegeli. After preliminary screening, only 18 polymorphic microsatellite loci were tested on a sample of 48 individuals. PCR for all loci was performed separately in a $25-\mu \mathrm{L}$ reaction volume containing $0.4 \mu \mathrm{M}$ of each primer, $0.2 \mathrm{mMdNTPs}, 2 \mathrm{mM} \mathrm{MgCl}_{2}, 1 \mathrm{X}$ PCR buffer, $1 \mathrm{U}$ Taq polymerase (Fermentas, Canada) and 50-100 ng DNA. Amplification was carried out with the following thermal profile: $94^{\circ} \mathrm{C}$ for $5 \mathrm{~min}$, followed by 35 cycles of $94^{\circ} \mathrm{C}$ for $45 \mathrm{~s}$, optimal annealing temperature (Table 1) for $45 \mathrm{~s}$, and $72^{\circ} \mathrm{C}$ for $45 \mathrm{~s}$, and a final extension step at $72^{\circ} \mathrm{C}$ for $10 \mathrm{~min}$. PCR products were separated on $6 \%$ denaturing polyacrylamide gels and vi- 
sualized by silver-staining. Allele sizes were estimated according to the pBR322/MspI marker.

The variability at each locus was measured in terms of number of alleles, expected heterozygosity and observed heterozygosity, and Hardy-Weinberg equilibrium (HWE) and linkage disequilibrium were tested using GENEPOP 4.0 (Raymond and Rousset, 1995). Null allele frequencies were calculated using Micro-Checker 2.2.3 (Van Oosterhout et al., 2004). The significant value for all diversity tests of significance was corrected by the sequential Bonferroni's procedure (Rice, 1989).

\section{RESULTS AND DISCUSSION}

The number of alleles per locus ranged from two to 15 (mean 5.7). The observed heterozygosity ranged from 0.0244 to 0.7660 (mean 0.4194 ), and expected heterozygosity ranged from 0.0244 to 0.8758 (mean 0.5002 ) (Table 1). Four loci departed significantly from HWE $(\mathrm{P}<0.05)$ after sequential Bonferroni's correction (adjusted $\mathrm{P}$ value $<0.00278)$. The observed departure from HWE is likely due to the evidence of "null" alleles (Py1-3, Py3-49, Py4-5, and Pyzj18). Three loci (Py3-8, Py3-9 and Pyzj8) showed significant pairwise linkage disequilibrium $(\mathrm{P}<0.05)$, after Bonferroni's correction. Nevertheless, these newly developed loci in combination with existing loci isolated from this species will enrich the microsatellite marker resources to assess population genetic structure and examine patterns of gene flow in the S. schlegeli.

Table 1. Characteristics of microsatellite loci in Sebastes schlegeli.

\begin{tabular}{|c|c|c|c|c|c|c|c|c|c|c|}
\hline Locus & Repeat motif & Primer sequence $\left(5^{\prime}-3^{\prime}\right)$ & Size range (bp) & $\mathrm{Ta}\left({ }^{\circ} \mathrm{C}\right)$ & A & $\mathrm{N}$ & $H_{\mathrm{O}}$ & $H_{\mathrm{E}}$ & $H_{\mathrm{O}} / H_{\mathrm{E}}$ & $\mathrm{P}_{\mathrm{HW}}$ \\
\hline Py1-3 $3^{+^{*}}$ & $(\mathrm{TG})_{14}$ & $\begin{array}{l}\text { F: ACTTTCACTGGTGCTTACT } \\
\text { R: CATGATCTGTCCTTTTCTC }\end{array}$ & $335-345$ & 57 & 6 & 46 & 0.1518 & 0.5721 & 0.266 & 0.0004 \\
\hline Py2-12* & $(\mathrm{AC})_{17}$ & $\begin{array}{l}\text { F: TGACCAACAGGAAAATAC } \\
\text { R: TTGAAAGATGACCCATTA }\end{array}$ & $310-403$ & 55 & 15 & 46 & 0.6957 & 0.8758 & 0.7944 & 0.0031 \\
\hline Py2-13 & $(\mathrm{AC})_{8}$ & $\begin{array}{l}\text { F: CATTCCACCTGTCTGTCC } \\
\text { R: AGATCGCCGATATTTGAT }\end{array}$ & $180-252$ & 55 & 6 & 48 & 0.625 & 0.6254 & 0.9994 & 0.0838 \\
\hline Рy3-6* & $(\mathrm{TG})_{12}$ & $\begin{array}{l}\text { F: ACGTATGTTGGCTGAAAC } \\
\text { R: TTTGGATAATGTGGCTTT }\end{array}$ & $213-230$ & 55 & 6 & 46 & 0.4130 & 0.5002 & 0.8257 & 0.0332 \\
\hline Ру3-8 & $(\mathrm{TG})_{10}$ & $\begin{array}{l}\text { F: CCCAGTAGGAGAAACTAAAC } \\
\text { R: AATGAATACACCCTCAGAAA }\end{array}$ & $260-275$ & 55 & 3 & 45 & 0.1778 & 0.1847 & 0.7913 & 0.0441 \\
\hline Рy3-9 & $(\mathrm{TG})_{6}$ & $\begin{array}{l}\text { F: CCCAGTAGGAGAAACTAAA } \\
\text { R: ATGAATACACCCTCAGAAA }\end{array}$ & $260-270$ & 55 & 3 & 48 & 0.2500 & 0.2432 & 1.028 & 0.6084 \\
\hline Рy3-10 & $(\mathrm{CA})_{8}$ & $\begin{array}{l}\text { F: TAAAGTGGTAGTTGGTGGTG } \\
\text { R: TTACCGAAAGTGTAGAAAGC }\end{array}$ & $259-270$ & 55 & 4 & 47 & 0.7447 & 0.7433 & 1.0019 & 0.3982 \\
\hline Рy3-27 & $(\mathrm{CA})_{13}$ & $\begin{array}{l}\text { F: ACAGATAAGTTATGCACCAA } \\
\text { R: TCGGGATAAGTAAGAGGA }\end{array}$ & $307-350$ & 55 & 8 & 47 & 0.7660 & 0.7463 & 1.0264 & 0.3737 \\
\hline Ру3-29 & $(\mathrm{TGG})_{5}$ & $\begin{array}{l}\text { F: TGCGGTGACTTATCCAGC } \\
\text { R: TCAACAAGGGAGCAAAGG }\end{array}$ & $315-346$ & 55 & 4 & 48 & 0.5833 & 0.4939 & 1.1810 & 0.9661 \\
\hline Рy3-41 & $(\mathrm{TG})_{8} \mathrm{AGT}(\mathrm{GA})_{5}$ & $\begin{array}{l}\text { F: CTGTTGGAGGGAGGTTAT } \\
\text { R: GTGTCTGGTTGAGCGAGT }\end{array}$ & $250-397$ & 55 & 8 & 48 & 0.5625 & 0.6428 & 0.8751 & 0.0954 \\
\hline Py $3-49^{\dagger *}$ & $(\mathrm{GT})_{16}$ & $\begin{array}{l}\text { F: ATCAATCACGCTCTTCTCA } \\
\text { R: GTCACGTAGATGGCTGAAA }\end{array}$ & $275-285$ & 55 & 6 & 47 & 0.3404 & 0.6383 & 0.5333 & 0.0000 \\
\hline Py4-2 & $(\mathrm{GT})_{5}$ & $\begin{array}{l}\text { F: TAACGCACTTACAGACAC } \\
\text { R: ATTATAGGATGAAGCCAC }\end{array}$ & $236-252$ & 59 & 4 & 48 & 0.5 & 0.4143 & 1.2069 & 1.0000 \\
\hline Py4-4 & $(\mathrm{CA})_{8}$ & $\begin{array}{l}\text { F: TCGGTGCAAGCAGGATGT } \\
\text { R: TAGCAGGACTGGAGGGAGG }\end{array}$ & $260-285$ & 55 & 5 & 48 & 0.5417 & 0.4581 & 1.1825 & 0.0146 \\
\hline Py4 $-5^{\dagger^{*}}$ & $(\mathrm{AC})_{21}$ & $\begin{array}{l}\text { F: ATGCACAGACAGAAATAC } \\
\text { R: TCGGATGATAATCAATAC }\end{array}$ & $215-245$ & 54 & 11 & 48 & 0.6042 & 0.8743 & 0.6911 & 0.0000 \\
\hline Py4-8 & $(\mathrm{CA})_{5}$ & $\begin{array}{l}\text { F: GTTCGCACAAACAGATAC } \\
\text { R: ACTGATAGCACCAGAATAA }\end{array}$ & $273-280$ & 55 & 2 & 48 & 0.0417 & 0.0412 & 1.0121 & 1.0000 \\
\hline Pyzj8 & $(\mathrm{TG})_{10} \ldots(\mathrm{TG})_{6}$ & $\begin{array}{l}\text { F: CCCAGTAGGAGAAACTAAA } \\
\text { R: CAAAACTAATGAATACACC }\end{array}$ & $270-285$ & 55 & 5 & 47 & 0.2553 & 0.2709 & 0.9424 & 0.3782 \\
\hline Pyzj16 & $(\mathrm{AC})_{5} \ldots(\mathrm{CT})_{9} \ldots(\mathrm{CA})_{5}$ & $\begin{array}{l}\text { F: ATTTCACAGCCTCGTTTAG } \\
\text { R: AGTAGGACAGGTGACTTCG }\end{array}$ & $338-343$ & 57.8 & 2 & 41 & 0.0244 & 0.0244 & 1.0000 & 1.0000 \\
\hline Pyzj18 $8^{* *}$ & $(\mathrm{GT})_{6} \ldots(\mathrm{AC})_{6} \ldots(\mathrm{TC})_{6}$ & $\begin{array}{l}\text { F: GTATTTGAGGGCATTTGT } \\
\text { R: CAATTAGTGATGAGGAAGAG }\end{array}$ & $290-302$ & 55.3 & 5 & 48 & 0.2708 & 0.6550 & 0.4134 & 0.0001 \\
\hline
\end{tabular}

$\mathrm{Ta}=$ optimized annealing temperature; $\mathrm{A}=$ number of alleles; $\mathrm{N}=$ number of individuals genotyped; $H_{\mathrm{O}}=$ observed heterozygosity; $H_{\mathrm{E}}=$ expected heterozygosity; $\mathrm{P}_{\mathrm{HW}}=$ Hardy-Weinberg probability; 'Locus deviated from HardyWeinberg equilibrium (adjusted $\mathrm{P}$ value $<0.00278$ ); *Locus may harbor null alleles (null allele frequency $>5 \%$ ). 


\section{ACKNOWLEDGMENTS}

Research supported by the National Natural Science Foundation of China ( $\# 40776097$ and \#31061160187) and Special Fund for Agroscientific Research in the Public Interest (\#200903005)

\section{REFERENCES}

An HS and Park JY (2009). Isolation and characterization of microsatellite markers for the heavily exploited rockfish Sebastes schlegeli, and cross-species amplification in four related Sebastes spp. Conserv. Genet. 10: 1969-1972.

Hu FG, Wang ZG, Zhang Y and Nie AH (2010). Research of parent Korean rockfish cultivating technique. Sci. Fish Farm. 4: $38-39$.

Kitada S, Shishidou H, Sugaya T, Kitakado T, et al. (2009). Genetic effects of long-term stock enhancement programs. Aquaculture 290: 69-79.

Li JL (2009). Fish resource enhancement abroad. Chin. Fish. Econ. 3: 111-123.

Litt M and Luty JA (1989). A hypervariable microsatellite revealed by in vitro amplification of a dinucleotide repeat within the cardiac muscle actin gene. Am. J. Hum. Genet. 44: 397-401.

Raymond M and Rousset F (1995). Genepop (version 1.2): population genetics software for exact test and ecumenicism. J. Heredity 86: 248-249.

Rice WR (1989). Analyzing tables of statistical tests. Evolution 43: 223-225.

Sambrook J and Russell DW (2001). Molecular Cloning. 3rd edn. Cold Spring Harbor, New York.

Schwartz MK, Luikart G and Waples RS (2007). Genetic monitoring as a promising tool for conservation and management. Trends Ecol. Evol. 22: 25-33.

Van Oosterhout C, Hutchinson WF, Wills DPM and Shipley P (2004). Micro-checker: software for identifying and correcting genotyping errors in microsatellite data. Mol. Ecol. Notes 4: 535-538.

Yoshida K, Nakagawa M and Wada S (2005). Multiplex PCR system applied for analyzing microsatellite loci of Schlegel's black rockfish, Sebastes schlegeli. Mol. Ecol. Notes 5: 416-418.

Zhan A, Bao ZM, Wang XL and Hu JJ (2005). Microsatellite markers derived from bay scallop Argopecten irradians expressed sequence tags. Fish Sci. 71: 1341-1346.

Zhan A, Hu J, Hu X, Zhou Z, et al. (2009). Fine-scale population genetic structure of Zhikong scallop (Chlamys farreri): do local marine currents drive geographical differentiation? Mar. Biotechnol. 11: 223-235. 